\title{
Estudio cinético del reformado seco de biogás
}

\author{
Daniel Zambrano Juca, Jaime Soler, Javier Herguido, Miguel Menéndez
}

\author{
Catalysis, Molecular Separations and Reactor Engineering Group (CREG) \\ Instituto de Investigación en Ingeniería de Aragón (I3A) \\ Universidad de Zaragoza, Mariano Esquillor s/n, 50018, Zaragoza, Spain. \\ Tel. +34-976762707, e-mail: 729450@unizar.es
}

\begin{abstract}
Se ha estudiado la cinética de las reacciones principales y la de desactivación para el reformado seco de biogás sobre un catalizador $5 \% \mathrm{Ni}-$ $10 \% \mathrm{Ce} / \mathrm{Al}_{2} \mathrm{O}_{3}$. Se seleccionaron en bibliografía 12 modelos mecanísticos a los cuales se ajustaron los datos experimentales mediante integración númerica. Para la pérdida de actividad del catalizador por formación de coque se utilizó un modelo de desactivación con actividad residual.
\end{abstract}

\section{Introducción}

La conversión del gas natural a gas de síntesis (syngas) es una importante opción como nueva fuente de energía. El syngas es una mezcla de $\mathrm{CO}+$ $\mathrm{H}_{2}$ que puede ser utilizado para obtener productos químicos de alto valor añadido tales como hidrocarburos, combustibles, compuestos oxigenados, etc. La principal desventaja que presenta este proceso para poder ser implementado a escala industrial es la formación de coque en el catalizador $\mathrm{y}$ por ende su desactivación. Para solucionar este problema, muchos trabajos han afrontado el reto de sintetizar catalizadores capaces de reducir la pérdida de actividad por formación de coque. Los catalizadores de níquel dopados con otros metales como el $\mathrm{Ce}$ [1] han mostrado un buen comportamiento en el proceso.

Para reducir la desactivación del catalizador se propone un proceso intensificado que involucra el uso de un reactor de lecho fluidizado de dos zonas (TZFBR) para la obtención de syngas con relaciones $\mathrm{CO} / \mathrm{H}_{2}$ cercanas a la unidad y la combinación con membranas de $\mathrm{Pd}-\mathrm{Ag}$ selectivas al hidrógeno (TZFBR-MR) para la obtención de hidrógeno de alta pureza [2].

Para una operación continua, este proceso requiere un adecuado balance entre la formación de coque y su eliminación. Por lo tanto se hace necesario realizar un estudio cinético del proceso para comprender el mecanismo de reacción y poder así determinar las condiciones óptimas de operación.

\section{Experimental}

El catalizador utilizado en la reacción se sintetizó mediante el método de impregnación a humedad incipiente con $5 \% \mathrm{Ni}-10 \% \mathrm{Ce}(\mathrm{wt} \%)$ soportados en $\mathrm{Al}_{2} \mathrm{O}_{3}$ (Sasol, Puralox ${ }^{\circledR S C C a}-150 / 200$ ), con un tamaño de partícula entre 106 y 180 m. Los experimentos catalíticos fueron realizados en un reactor de cuarzo de lecho fijo, para lo cual se dispuso de una planta a escala de laboratorio. Se realizaron estudios previos para asegurar que la reacción esté siendo controlada por la cinética y evitar problemas de control difusional.

Los experimentos se realizaron a temperaturas entre $475-550^{\circ} \mathrm{C}$ a presión atmosférica. Los gases reactantes $\left(\mathrm{CO}_{2}+\mathrm{CH}_{4}\right)$ fueron alimentados mediante controladores de flujo másico con diferentes ratios $\mathrm{CH}_{4}: \mathrm{CO}_{2}: \mathrm{N}_{2}$. El lecho catalítico estuvo conformado por $0,5 \mathrm{~g}$ de catalizador y una cantidad igual de $\mathrm{SiC}$, utilizado como inerte. El tiempo de reacción de cada experimento fue de 4 horas y los gases de salida se analizaron cada 20 minutos mediante cromatografía de gases. Tras cada experimento, el catalizador fue regenerado con una corriente de $\mathrm{O}_{2}$ a $600^{\circ} \mathrm{C}$ para eliminar el coque formado y luego reducido en una corriente de $\mathrm{H}_{2}$ a $700^{\circ} \mathrm{C}$ durante 3 horas.

Para el modelado cinético se consideraron las reacciones de reformado seco de metano, reacción water gas shift inversa y descomposición de metano, así como la desactivación del catalizador. El ajuste de los datos experimentales a los modelos matemáticos se realizó mediante integración númerica de la ecuación de diseño del reactor de flujo pistón y minimización de una función objetivo utilizando para ello el algoritmo de LevenbergMarquardt y una aplicación desarrollada en MatLab $^{\circledR}$. Para determinar el modelo estadísticamente significante y termodinámicamente consistente con los datos experimentales se utilizaron factores de comparación de modelos como el AIC, BIC y test de Fisher. 


\section{Resultados}

Para la reacción de reformado se consideraron un total de 12 modelos de tipo mecanístico obtenidos de la bibliografía, mientras que para la desactivación de catalizador se consideraron los modelos de desactivación de primer orden y desactivación con actividad residual. Teniendo en cuenta los factores de comparación de modelos se pudo determinar que el modelo cinético de tipo Stepwise y el modelo de desactivación con actividad residual (Tabla 1) fueron los de mejor ajuste para los datos experimentales.

Tabla 1. Modelos para el reformado seco de biogás

\begin{tabular}{|c|}
\hline Modelo cinético (Stepwise) [3] \\
\hline$-r=\frac{k_{1} P_{\mathrm{CH}_{4}}}{\left(1+\frac{P_{\mathrm{CO}}^{2}}{P_{\mathrm{CO}_{2}} K_{c(s)}}\right)}\left(1-\frac{P_{\mathrm{CO}}^{2} P_{\mathrm{H}_{2}}^{2}}{P_{\mathrm{CH}_{4}} P_{\mathrm{CO}_{2}} K_{\text {eq }}}\right)$ \\
\hline Modelo de desactivación con actividad residual [4] \\
\hline$a=\frac{\varphi_{d}}{\left(\varphi_{d}+\varphi_{r}\right)} e^{-\left(\varphi_{d}+\varphi_{r}\right) * t}+\frac{\varphi_{r}}{\left(\varphi_{d}+\varphi_{r}\right)}$ \\
\hline
\end{tabular}

La Fig. 1 muestra el ajuste del modelo cinético a los datos experimentales a tiempo cero. En la Fig.2 se observa la desactivación inicial del catalizador que luego tiende a estabilizarse para mantener una actividad residual en los experimentos con exceso de metano en la alimentación.

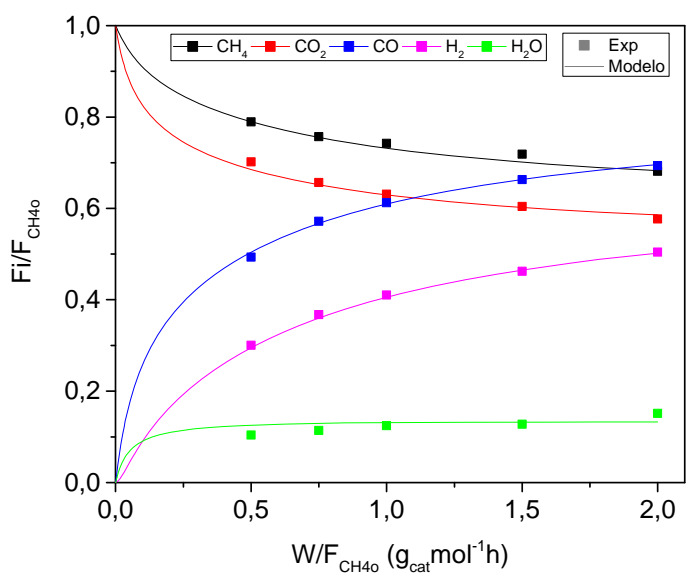

Fig. 1 Flujos iniciales $(t=0)$ vs tiempo espacial. Temperatura $550{ }^{\circ} \mathrm{C}$ y relación $\mathrm{CH}_{4}: \mathrm{CO}_{2}: \mathrm{N}_{2}=1: 1: 0.5$

\section{Conclusiones}

Se ha realizado un estudio cinético para el proceso de reformado seco de biogás con un catalizador Ni$\mathrm{Ce} / \mathrm{Al}_{2} \mathrm{O}_{3}$ teniendo en cuenta la reacción de reformado así como la desactivación del catalizador. Los datos obtenidos muestran que la desactivación del catalizador está relacionada fundamentalmente con la presión parcial de metano, mientras que es inhibida por la presión parcial del dióxido de carbo, el cual gasifica el coque formado.

\section{REFERENCIAS}

[1]. LAOSIRIPOJANA, N., SUTTHISRIPOK, W. and ASSABUMRUNGRAT, S. Synthesis gas production from dry reforming of methane over $\mathrm{CeO} 2$ doped $\mathrm{Ni} / \mathrm{Al2O} 3$ : Influence of the doping ceria on the resistance toward carbon formation. Chemical Engineering Journal. 2005. Vol. 112, no. 1-3, p. 1322. DOI 10.1016/j.cej.2005.06.003.

[2]. UGARTE, P., DURÁN, P., LASOBRAS, J., SOLER, J., MENÉNDEZ, M. and HERGUIDO, J. Dry reforming of biogas in fluidized bed: Process intensification. International Journal of Hydrogen

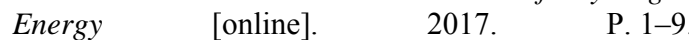
DOI 10.1016/j.ijhydene.2016.12.124.

[3]. EL SOLH, T, JAROSCH, $\mathrm{K}$ and DE LASA, $\mathrm{H}$. Catalytic dry reforming of methane in a CREC riser simulator kinetic modeling and model discrimination. Industrial \& Engineering Chemistry Research. 2003. Vol. 42, no. 12, p. 2507-2515. DOI 10.1021/ie020749d.

[4]. MONZÓN, A., ROMEO, E. and BORGNA, A. Relationship between the kinetic parameters of different catalyst deactivation models. Chemical Engineering Journal. 2003. Vol. 94, no. 1, p. 19-28. DOI 10.1016/S1385-8947(03)00002-0.

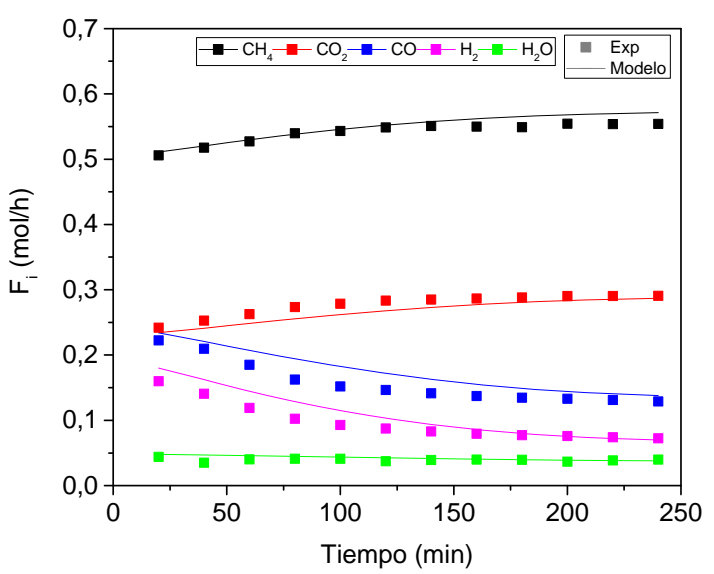

Fig. 2 Flujos vs tiempo de operación. Temperatura $525{ }^{\circ} \mathrm{C}$ y relación $\mathrm{CH}_{4}: \mathrm{CO}_{2}: \mathrm{N}_{2}=1: 0.6: 0.4 \mathrm{~W} / \mathrm{F}=0.8\left(\mathrm{~g}(\mathrm{cat}) \mathrm{mol}^{-1} \mathrm{~h}\right)$ 